\title{
Importancia del potasio en el líquido de baño para prevenir hipopotasemias
}

\author{
$\mathbf{M}^{\mathrm{a}}$ Carmen Salvador Lengua, $\mathbf{M}^{\mathrm{a}}$ Jose Folch Morro, Jorge Bort Castello, Alexandra Mallol Domínguez, \\ Vicente Cerrillo García, Elena Renau Ortells
}

\section{Servicio de hemodiálisis. Hospital General. Castellón}

\section{Introducción:}

Las alteraciones del potasio $\left(\mathrm{K}_{+}\right)$son de las más frecuentes en hemodiálisis (HD). La hipopotasemia se manifiesta por una disminución de las concentraciones plasmáticas de $\mathrm{K}+$ inferior a $3.5 \mathrm{mEq} / \mathrm{l}$ (miliequivalentes/litro), considerándose hipopotasemia grave $\mathrm{K}+$ inferior $2.5 \mathrm{mEq} / \mathrm{l}$. Es de vital importancia que enfermería esté atenta y vigilante a estas alteraciones que se producen en el desequilibrio hidroelectrolítico. En nuestra unidad de diálisis tuvimos la posibilidad de adquirir un gasómetro y poder personalizar el tratamiento de hemodiálisis en función del K+ inicial tras la determinación de una gasometría venosa. Por eso decidimos realizar un estudio acerca de las variaciones del $\mathrm{K}+$ durante la sesión de HD. Nuestros objetivos han ido encaminados a: evitar hipopotasemias graves durante el tratamiento dialítico, individualizar cada sesión para favorecer la tolerancia en la diálisis y mejorar la calidad asistencial. Ha sido un estudio descriptivo longitudinal. Los pacientes estudiados pertenecen a la sala de agudos durante los meses de enero y febrero de 2012. A cada paciente se le realizaba una gasometría pre y post hemodiálisis. Todos los pacientes iniciaban la diálisis con un $\mathrm{K}+1,5 \mathrm{mEq} / \mathrm{l}$ en el baño. Según el valor del $\mathrm{K}+$ en la gasometría variábamos la cantidad de $\mathrm{K}+$ en el baño. En 46 ocasiones se registró un ritmo sinusal, en un caso obtuvimos una arritmia y en otro una fibrilación auricular crónica. En 32 casos se administró en el baño $\mathrm{K}+$ $1,5 \mathrm{mEq} / \mathrm{l}$, en 22 casos se administró en el baño $\mathrm{K}+2 \mathrm{mEq} / \mathrm{l}$ y en 30 casos se administró en el baño $K+3 \mathrm{mEq} / \mathrm{l}$. Todos los pacientes no estaban monitorizados por falta de medios, pero se tenía en cuenta a los pacientes cardíacos. Aunque en todos los casos los pacientes terminaron con un potasio sérico bajo, en ningún caso se observó hipopotasemia grave. Este tema en cuestión ha presentado dificultades en la búsqueda de bibliografía. La gasometría solo se realizaba a los pacientes agudos, puesto que son más inestables, pero tene- mos la opción de realizarla a cualquier paciente de nuestro servicio, cosa que nos es muy útil en el funcionar del día a día. La realización de la gasometría previa a la conexión es una actividad que ha asumido el personal de enfermería, sin que suponga una sobrecarga de trabajo. Mediante este estudio se confeccionó una tabla, con la ayuda de los nefrólogos de la unidad, para poder unificar los criterios de utilización del $\mathrm{K}+$ en el baño. Remarcar que no obtuvimos ninguna alteración cardíaca intradiálisis. Gracias a la posibilidad de realizar una GSV en cualquier momento de la diálisis y el hecho de poder monitorizar al paciente nos facilita detectar precozmente una hipopotasemia durante la sesión realizando un tratamiento personalizado y controlado. Consiguiendo también aumentar la calidad asistencial. Una vez realizada la tabla para unificar los criterios en la utilización del K+ comparamos únicamente los pacientes del estudio que se ajustaban a dicha tabla.

\section{Referencias Bibliográficas}

1. P. Aljama García, M. Arias Rodríguez, C. Caramelo Díaz, J. Egido de los Rios y S. Lamas Peláez. Nefrología Clínica, $3^{a}$ edición. Pág 61-67. 2009.

2. P. De Sequera Ortiz, R. Alcázar Arroyo y M. Albalate Ramón. Nefrología al día II. Trastornos electrolíticos ácido-base. Trastornos del potasio. Pág 3. Nov 2010.

3. R. Alonso Nates, R. Pelayo Alonso. Manual de Enfermería Nefrológica. Pág 205.0ct 2012.

4. Smelle WS. Spurius hyperkalaemia. BMJ. 2007; 334: 693-5. 5. Zehnder C, Gutzwiller JP, Huber A, Schindler C, Schneditz D. Low-potassium and glucose free dialysis maintains urea but enhances potasium removal. Nephrol Dial Transplant. 2001; 16: 78-84. 